\title{
Thelper 2 inflammatory markers are associated with recurrence in chronic rhinosinusitis with nasal polyps after endoscopic sinus surgery*
}

\author{
Geoffrey Mortuaire ${ }^{1,2}$, Isabelle Gengler', Chloé Carpentier', \\ Claire Szymanski', Cécile Chenivesse ${ }^{3}$, Guillaume Lefèvre ${ }^{1,2,4}$ \\ Otorhinolaryngology - Head and Neck Department, Huriez Hospital, CHU Lille, France \\ 2 Lille Inflammation Research International Center - Inserm U995, University of Lille, France \\ ${ }^{3}$ Pneumology and Allergology Department, Calmette Hospital, CHU Lille, 59000 Lille, France \\ ${ }^{4}$ Medicine and Clinical Immunology Department, National Reference Center of Hypereosinophilic Syndromes, CHU Lille, France
}

Rhinology 58: 5, $444-450,2020$

https://doi.org/10.4193/Rhin19.439

*Received for publication:

December 5, 2019

Accepted: February 28, 2020

\begin{abstract}
Background: Despite maximum medical treatment and endoscopic sinus surgery (ESS), chronic rhinosinusitis with nasal polyps (CRSwNP) can require revision surgery. With a growing literature on the diversity of cytokine inflammation patterns in CRSwNP, an endotype-driven approach could lead to the identification of cytokine profiles that predict recurrence.
\end{abstract}

Methods: A monocentric longitudinal study was carried out until June 2019 following CRSwNP patients who underwent surgery for the first time between December 2010 and January 2012. The biomarker profiles were established on blood and nasal secretions at the time of the first surgery (Interleukin (IL)-5, $\lg \mathrm{E}$, IgA, eosinophilic cationic protein (ECP) and eosinophilic- derived neurotoxin (EDN)). Profiles were compared between the patients still controlled by medical treatment and the patients requiring revision surgery during the course of the follow-up period.

Results: Among the 48 patients initially enrolled in our study, 8 required revision surgery (16,7\%). Clinical features (asthma, allergy, aspirin intolerance, active smoking) and levels of blood markers measured at the time of the first surgery were comparable between the 2 groups of patients. Levels of IL-5, IgE and ECP in nasal secretions were significantly increased in the group of patients needing revision surgery.

Conclusions: Based on simple approach of nasal secretions sampling, we showed that a predominant Thelper 2 proteins expression profile can be associated with recurrent CRSWNP after ESS. Initial immunoprofiling in CRSwNP disease may contribute to better predict the therapeutic response to optimal medical and surgical treatment, and help define the role of innovative targeted treatment, beside corticosteroids and ESS.

Key words: endotype, eosinophils, interleukin-5, nasal polyposis, refractory

\section{Introduction}

Chronic rhinosinusitis with nasal polyps (CRSwNP) is a major concern with a prevalence of $2-4 \%$ in Western countries ${ }^{(1)}$. This disease is often associated with comorbidities such as asthma or nonsteroidal anti-inflammatory drugs-exacerbated respiratory disease (N-ERD). Nasal polyposis has a substantial impact on social and physical quality of life. It also creates a significant economic burden, secondary to absenteeism from work and cost of treatment ${ }^{(1-4)}$.

CRSwNP has been widely investigated. It is characterized by a diverse clinical phenotype with mixed pathophysiologies leading to various inflammatory profiles. In European population, CRSwNP is mainly considered to be orchestrated by Thelper (Th) 2 cells, with interleukin (IL)- 5 as the major cytokine, resulting in an eosinophilic type of inflammation, associated with local lgE production ${ }^{(5)}$. However, the extent of eosinophilia can be quite 
variable as some patients show a Th1/Th17 polarization, characterized by low eosinophilic cationic protein (ECP) levels, the main degranulation product of eosinophils involved in tissue remodeling ${ }^{(6,7)}$. Tomassen et al. characterized 10 potential CRS endotypes based on cluster analysis of different inflammatory mediators within surgical tissue specimen ${ }^{(8)}$. In this study, 5 out of the 10 clusters described belong to Th2 inflammation.

All of these features make the treatment of CRSwNP very challenging and require a bespoke therapeutic approach to fully address different patients' immunoprofiles ${ }^{(2)}$. So far, endoscopic sinus surgery (ESS) remains the treatment of choice when the first line of medical treatment with corticosteroid has failed. Regardless of the surgical technique, some patients tend to be poorly controlled leading to subsequent revision surgeries. A recent cohort study of patients with CRSwNP reported an overall recurrence rate of $40 \%$ after ESS ${ }^{(9)}$. Several studies based on the analysis of surgically excised nasal mucosa have been published to identify specific inflammatory profiles associated with revision surgeries ${ }^{(6,10)}$. Diagnostic methods using surgical specimens can be time-consuming and difficult to apply in a routine biological workup.

In this study, we investigated levels of biomarkers of interest, in blood and nasal secretions, in a cohort of patients with refractory CRSwNP who underwent ESS for the first time. With a standardized longitudinal follow-up of this cohort, we aimed to identify a specific immune profile associated with increased risk of revision surgery.

\section{Material and methods}

\section{Population}

A single-center prospective longitudinal study included patients with CRSwNP diagnosed according to the 2012 European position paper on rhinosinusitis and nasal polyps guidelines (1), aged over 18 years, resistant to medical treatment, for whom endoscopic sinus surgery (ESS) was indicated. Refractory CRSwNP was defined by persistent subjectively disabling symptomatology (visual analogic scale for symptom discomfort over ${ }^{(7)}$, with 10 = maximum discomfort) despite maximum-dose local corticotherapy and three 10-day courses of oral corticosteroids at 1mg/ $\mathrm{kg} /$ day prednisone equivalent over a period of 1 year. Exclusion criteria comprised CRSwNP associated with mucociliary pathology, immune deficiency or autoimmune disease. The patients were enrolled from December 2010 to January 2012 for clinical data collection and biological sampling.

Written informed consent was obtained ahead of any analysis of serum or nasal secretion. Exploration was performed under the SHE (Hyper-Eosinophilia Syndrome) study, approved by the local review board (North West Ethical Council, n² 2009-A00314-53).

\section{Clinical data}

Preoperative clinical data comprised: age, gender, smoking status, and history of sinonasal surgery. Atopy was identified on concordant interview and skin prick tests data. Asthma and bronchial hyper-reactivity were systematically screened for on interview, respiratory function tests and methacholine challenge, as appropriate. N-ERD was clinically assessed based on respiratory symptoms reported when using non-steroidal antiinflammatory drugs, during patient interview.

CRSWNP severity was assessed in terms of preoperative symptom intensity on a visual analog scale (VAS: 0-10cm, with $10=$ maximal functional discomfort). Polyp size was measured on Lildholdt score ${ }^{(11)}$ on preoperative endoscopy without mucosal retraction. A mean score for both sides was reported (from 0 to 3). Sinonasal CT scan without contrast was performed within 2 weeks before the first surgery, for the purposes of 3D surgical navigation during the procedure, and to assess the Lund-Mackay score (from 0 to 24) (12).

Patients were required to interrupt any local or general corticosteroid treatment at least 1 month before surgery. Only saline nasal lavage was recommended.

\section{Biological sampling}

Blood samples were collected before surgery, in BD Vacutainer ${ }^{\mathrm{TM}}$ EDTA tubes (BD Medical, Franklin Lakes, NJ, USA). Polynuclear eosinophils were assayed in blood by routine clinical cell count. Nasal secretions were collected in the operating room, just before the start of the procedure. Two $1.27 \times 2.54 \mathrm{~cm}$ cotton swabs (Codamn, Raynham, MA, USA) were introduced in each nasal cavity for 5 minutes, immersed in PBS (phosphate-buffered saline) for $1 \mathrm{~h}$ at $4^{\circ} \mathrm{C}$, then centrifuged at $14,000 \mathrm{rpm}$ for $8 \mathrm{~min}$ to eliminate cellular components. Supernatants for inflammation marker evaluation were aliquoted after Bradford protein assay, and kept at $-80^{\circ} \mathrm{C}$.

Products of eosinophil degranulation: ECP, EDN (eosinophil-derived neurotoxin), and IL-5 were assayed in serum and nasal secretions, using MBL International (Nagoya, Japan), R\&D Systems (Minneapolis, MN, USA) and Bio-Rad (Hercules, CA, USA) ELISA kits. Detection thresholds were $0.62 \mathrm{ng} / \mathrm{ml}$ for ECP and EDN, and $0.29 \mathrm{pg} / \mathrm{ml}$ for IL-5.

Total $\lg \mathrm{E}$ and total $\lg \mathrm{A}$ were respectively assayed in serum and nasal secretions by Phadia ${ }^{\mathrm{TM}}$ immunoCAP test (Thermo Scientific, Waltham, MA, USA) and SPAplus ${ }^{\mathrm{TM}}$ analyzer (Binding Site, Birmingham, UK).

\section{Patients follow-up}

After surgery, the cohort of patients was followed by the first author at 3 months, 6 months and every 12 months postoperatively until June 2019. They were all treated with topical steroid

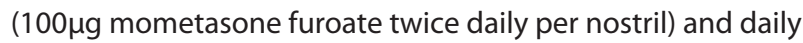
nasal saline irrigation. A maximum of three 10-day courses of oral corticosteroids at $1 \mathrm{mg} / \mathrm{kg} /$ day prednisone equivalent over a period of 1 year was allowed in uncontrolled patients. Refractory 
Table 1. Demographics characteristics of the patients at the first surgery.

\begin{tabular}{|c|c|c|c|c|}
\hline & & $C$ group $(n=40)$ & $R$ group (n=8) & $\mathbf{p}$ \\
\hline Sex (male) & & $25(62.5)$ & $5(62.5)$ & 0.647 \\
\hline Mean age (yr) & & $51.0(13.4)$ & $41.6(13.7)$ & 0.061 \\
\hline Atopy & & $8(20)$ & $1(12.5)$ & 0.530 \\
\hline Asthmatic & & $20(50)$ & $6(75)$ & 0.183 \\
\hline N-ERD & & $7(17.5)$ & $4(50)$ & 0.068 \\
\hline Mean Lilholdt score (0-3) & & $2.3(0.58)$ & $2.2(0.61)$ & 0.745 \\
\hline Lund-Mackay score (0-24) & & $18.7(3.2)$ & $19.5(4.0)$ & 0.365 \\
\hline VAS symptoms $(0-10 \mathrm{~cm})$ & & $6.9(2.0)$ & $8.1(1.3)$ & 0.160 \\
\hline \multirow{2}{*}{ Smoking } & Ex-smoker & $0(0)$ & $0(0)$ & \\
\hline & Current smoker & $5(12.5)$ & $1(14)$ & 0.742 \\
\hline Follow-up period (yr) & & $7.0(1.3)$ & $6.7(1.4)$ & 0.609 \\
\hline \multirow{2}{*}{ Symptoms duration (yr) } & Onset to 1 st surgery in our institution & $11.7(10.2)$ & $11.4(9.4)$ & 0.923 \\
\hline & 1 st to 2 nd surgery in our institution & NA & $5.0(2.4)$ & \\
\hline
\end{tabular}

Numerical data are expressed as mean ( \pm standard deviation). Categorical data are expressed as number (\%). VAS: visual analogic scale, N-ERD: nonsteroidal anti-inflammatory drugs-exacerbated respiratory disease.

CRSwNP after the first surgery was again defined by persistent subjectively disabling symptomatology (VAS over 7) despite maximum-dose local corticotherapy and the above mentioned systemic steroid protocol. When those criteria were fulfilled, revision surgery based on a systematic reopening of all sinuses was proposed. During this period, patients who underwent revision surgeries due to failed maximal medical treatment after the first surgery in our institution were defined as refractory to surgical treatment (R group). Those who were still controlled by medical treatment after the first surgery were defined as controlled (C group).

Clinical data and biological biomarkers obtained at the moment of the first surgery were compared between the R group and the C group.

\section{Statistical analysis}

Data were anonymized and entered in Microsoft Excel ${ }^{\mathrm{TM}}$ file. Statistical analysis used SPSS ${ }^{\mathrm{TM}}$ software v15.0 (SPSS Inc., Chicago, IL, USA). Means and standard deviations were reported for descriptive variables. $\mathrm{Chi}^{2}$ or Fisher exact tests were used to compare frequencies. Given the relatively small patient group, the non-parametric Mann-Whitney test was used to compare means between non-matched groups. Odds ratios (ORs) were calculated using logistic regression analysis. The significance level was set at $a=0.05$.

\section{Results}

\section{Clinical data}

Forty-eight patients were included over the study period: 30 male subjects, 18 female subjects with a mean age of $49.5 \pm$
13.8 years at the moment of the first surgery in our institution. None of them was previously treated with surgery in another institution. The mean follow-up duration after the first surgery in our institution was $6.8 \pm 1.3$ years for all the patients. During the study period, 8 patients (16.7\%) required revision surgery $(R$ group). The mean time to second surgery in the R group was 5.0 years (Table 1).

Demographic details of subjects' characteristics at the moment of the first surgery in our institution are shown in Table 1. Both groups (C group and R group) were comparable in terms of age, gender ratio, atopy (positive skin-prick test) and smoking habit. Asthma and N-ERD were more frequent in the $\mathrm{R}$ group without reaching a significant statistical level. Symptoms score based on VAS, polyp score and CT score were also comparable between the two groups.

No difference was observed between the $\mathrm{C}$ group and the $\mathrm{R}$ group in terms of follow-up duration (respectively 7.0 and 6.7 years).

\section{Biological data}

Concentrations of eosinophils, IgE, IgA, ECP, EDN and IL-5 in blood or in nasal secretions at the first surgery in our institution are summarized in Table 2 . For all biomarkers measured in blood, there was no statistically significant difference in the level of expression between both groups (Figure 1), whereas total lgE, ECP and IL-5 in nasal secretions were significantly up-regulated in CRSwNP patients needing revision surgery (R group) (Figure 2).

A binary logistic regression model was built using the clinical characteristics and biomarkers of our population to calculate 
Table 2. Biological characteristics of the patients at the first surgery.

\begin{tabular}{|c|c|c|c|c|}
\hline & & $C$ group ( $n=40)$ & $R$ group $(n=8)$ & $\mathbf{p}$ \\
\hline \multirow[t]{5}{*}{ Blood } & $\mathrm{EO}(/ \mathrm{mm} 3)$ & $652(180)$ & $662(574)$ & 0.784 \\
\hline & Total lgE (KU/I) & $141(190)$ & 239(188) & 0.132 \\
\hline & Total IgA (KU/I) & $1.43(0.49)$ & $1.45(0.46)$ & 0.976 \\
\hline & $\mathrm{ECP}(\mathrm{ng} / \mathrm{ml})$ & $39(39)$ & $40(75)$ & 0.177 \\
\hline & EDN (ng/ml) & $62(30)$ & $69(44)$ & 0.812 \\
\hline \multirow[t]{5}{*}{ Nasal secretions } & Total lgE (KU/I) & $19.6(20.3)$ & $57.2(56.5)$ & 0.05 \\
\hline & Total IgA (KU/I) & $0.14(0.08)$ & $0.14(0.09)$ & 0.881 \\
\hline & $\mathrm{ECP}(\mathrm{ng} / \mathrm{ml})$ & 156(137) & $555(415)$ & 0.04 \\
\hline & EDN (ng/ml) & $201(82)$ & $593(728)$ & 0.5 \\
\hline & IL-5 (pg/ml) & $14.0(14.5)$ & $91.3(62.5)$ & 0.02 \\
\hline
\end{tabular}

Numerical data are expressed as mean ( \pm standard deviation). ECP: eosinophil cationic protein; EDN: eosinophil-derived neurotoxin; EO: eosinophils; IL-5: interleukin-5.

the ORs for revision surgery. The only positive OR for revision surgery was found with high levels of IL-5. However, the $95 \%$ confidence interval included one, making this OR not statistically significant.

\section{Discussion}

Although CRSwNP has been considered and treated as one single clinical disease entity, biological evidence gathered during the last ten years highlights that CRSwNP should be considered a group of different immunologic entities. To fully implement precision medicine into daily practice, disease management for CRSwNP needs to be complemented with inflammatory endotyping ${ }^{(7)}$. Recent introduction of monoclonal antibodybased therapeutics that target IL-4/IL-13 and IL- 5 have shown great promise for managing recalcitrant CRSWNP and may be soon considered valid alternatives to endoscopic sinus surgery ${ }^{(13,14)}$. Regarding recurrent CRSwNP needing revision surgery, classifications based on clinical phenotypes (i.e. asthma, N-ERD or allergy) are not always relevant to predict optimal disease control (2). In our study, we hypothesized that the phenotypes based on clinical symptoms and respiratory comorbidities were not always useful to clearly differentiate patients with medically controlled CRSwNP from patients requiring revision surgery. Even if our conclusions are based on a small population, results of nasal endoscopy and sinus CT scanning were similar in the two groups of patients. As previously described, there would be no major role for allergy as a predictive factor for revision surgery ${ }^{(15)}$.

Endotype-driven treatment requires a clinical applicable approach with easily measurable biological markers ${ }^{(2)}$. In that perspective, we chose to focus our analysis on blood and nasal secretions biomarkers, readily accessible during patient examination. We previously demonstrated in our population that CRSwNP is linked to a Th2 type inflammatory pattern. This immune profile is strongly associated with comorbid asthma ${ }^{(16)}$. Based on mucosal level of IL-5 and IL-13, Turner et al. showed that Th2 biomarkers were able to predict CRSwNP severity and clinical characteristics ${ }^{(17)}$. Herein, a monocentric longitudinal study was carried out to improve the homogeneity of the results. The same surgical technique and postoperative steroid medication protocol were implemented to reduce biases in the selection of patients needing revision surgery after a first ESS. Moreover, revision surgery indication was always applied according to the same clinical and therapeutic criteria described in the methods section. Our rate of surgical revision (16.7\%) over a follow-up period of 6.8 years is in accordance with published data ${ }^{(9)} 9$. In a cohort study of 549 patients with nasal polyposis, Mendelsohn et al. described a 5 -year recurrence rate of $25 \%$ in patients with concomitant asthma ${ }^{(18)}$.

Here, we show that recurrent CRSwNP needing revision surgery could exhibit a more predominant Th2 type inflammation with increased concentrations of IL-5, ECP and IgE in nasal secretions. Our results are in line with previously published studies. In a less practicable approach based on polyp tissue homogenates, Van Zele et al. found increased concentrations of specific lgE to staphylococcal super antigens in recurrent CRSwNP whereas non-recurrent CRSwNP exhibited a mixed Th1-Th2 and Th17 pattern with higher levels of IFN- ${ }^{\left({ }^{(6)}\right.}$. The same results were described by Wei et al. in 78 patients undergoing functional endoscopic sinus surgery who were followed for 8 years and classified into recurrent and non-recurrent groups ${ }^{(19)}$. In our study, serum levels of IgE and other markers were not discriminant between patients with controlled and recurrent CRSwNP after a first surgery. Blood Eosinophilia as a landmark of CRSwNP could not be as relevant to predict the risk of revision surgery in our population. The definition of eosinophilic CRSWNP is not 

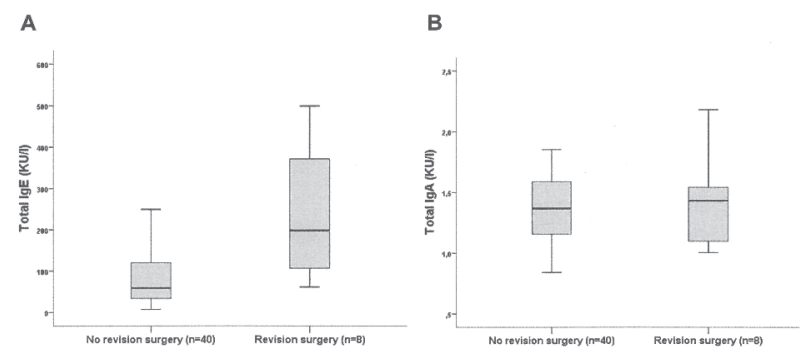

C
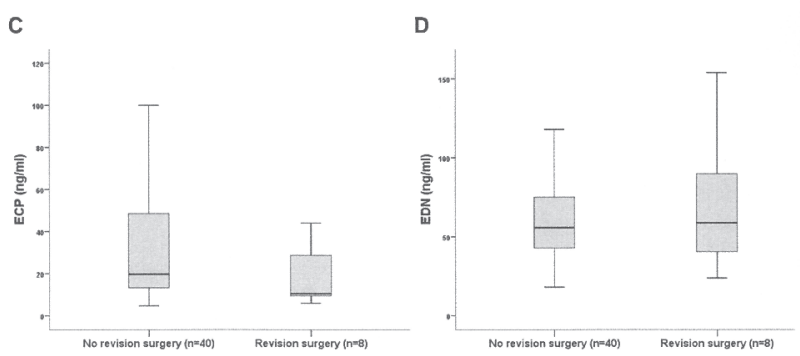

E

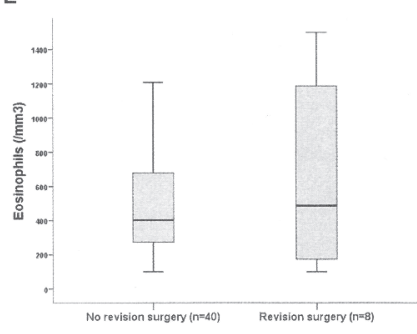

Figure 1. Expression of inflammatory mediators at the first surgery in blood samples. Proteins levels of (A) Total lgE, (B) Total IgA, (C) eosinophil cationic protein (ECP), (D) eosinophil-derived neurotoxin (EDN) and (E) eosinophil counts were comparable in the two groups of patients. Concentrations of Total IgE and Total IgA were expressed in $\mathrm{KU}$ per liter. Concentrations of ECP and EDN were expressed in nanograms per milliliter. Eosinophil count was expressed in number of cells per mm3. Statistical significance $(p<0.05)$ was determined by Mann-Whitney test Plots represent medians and interquartile ranges.

standardized, and no consensus exists regarding the level of eosinophilia that should define a given patient's profile ${ }^{(20)}$. In a retrospective analysis of 387 patients followed over 24 months for polyp recurrence, Lou et al. showed that blood eosinophil counts did not predict the surgical response to the same degree that tissue eosinophil counts did ${ }^{(21)}$. On the contrary, in a retrospective analysis of 1716 CRS treated with ESS, Tokunaga et al. showed that the recurrence-free rate was inversely associated with the number of eosinophils in nasal polyps measured on high-power field ${ }^{(22)}$. As previously observed by De Schryver et al. recognizing local IgE production in the absence of systemic $\lg \mathrm{E}$ higher concentration, we assume that a more accurate approach to determine the severity of inflammation at the target organ level is to evaluate markers locally ${ }^{(23)}$. A method based on nasal secretion measurement is noninvasive and less time consuming than polyp collection or supernatant tissue extraction. Previous
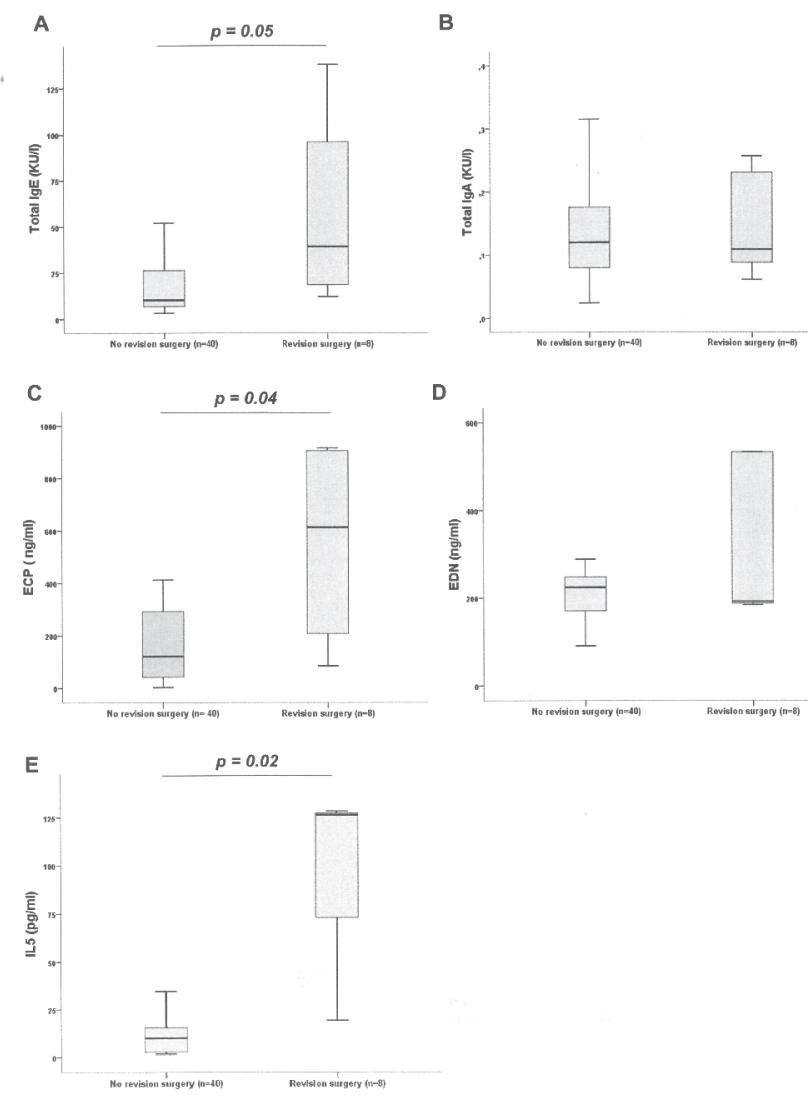

Figure 2. Expression of inflammatory mediators at the first surgery in nasal secretions. Proteins levels of $(A)$ Total $\lg E,(C)$ eosinophil cationic protein (ECP) and (E) interleukin-5 (IL-5) were increased in patients with revision surgery. Proteins levels of (B) Total IgA, (D) eosinophil-derived neurotoxin (EDN) were comparable in the two groups of patients. Concentrations of Total IgE and Total IgA were expressed in $\mathrm{KU}$ per liter. Concentrations of ECP and EDN were expressed in nanograms per milliliter. Concentrations of IL-5 was expressed in picograms per milliliter. Statistical significance $(p<0.05)$ was determined by Mann-Whitney test. Plots represent medians and interquartile ranges.

work has shown that the local sinonasal environment appears to influence specific inflammatory mediators expression leading to potential discrepancies in biomarker levels measured in tissue extracted from different regions, such as the inferior turbinate or the ethmoidal sinus mucosa ${ }^{(24)}$. Another study found that nasal secretions directly collected on sponges are a good surrogate for estimating polyp tissue inflammation ${ }^{(25)}$. We believe that nasal secretion sampling in CRS can be routinely applied even in non-surgical cases to further differentiate CRS clusters with diverse inflammatory mechanisms. Using logistic regression analysis, we did not show significant ORs for revision surgery when considering IgE, ECP and IL-5. The same results were observed by Van Zele et al. in 21 non recurrent CRSwNP and 15 recurrent CRSwNP after a first surgery ${ }^{\left({ }^{6}\right)}$. Ongoing recruitment of patients is required to improve the statistical power of the study on a larger population. 
The Th2 inflammatory endotype is the most common one found in patient populations in Europe and in the US, with high IL-5 eosinophilic CRSwNP observed in $83 \%$ of all patient population $(2,26,27)$. Nevertheless, the heterogeneity of CRSwNP inflammation pathways (Th1, Th2 and Th17) is well documented and recent studies suggest variable inflammatory endotypes based on geographic locations and ethnic differences ${ }^{(8.28)}$. Wang et al. showed in a multicenter study performed in Europe, Asia and Oceania that polyps display a predominant low IL-5 neutrophilic inflammatory pattern in South Chinese patients ${ }^{(26)}$. In the same study, $17 \%$ of patients from Benelux also showed high Th 17 cytokines expression without IL-5 expression suggesting the presence of immunologic differences, within the same European area. A case-control study performed in 156 Korean patients with nasal polyposis showed that neutrophil-related mediators (myeloperoxidase, IL-8), Th17 associated cytokines (IL-17A and IL-23) and IgA were up-regulated in patients with refractory CRSwNP ${ }^{(10)}$. Other studies showed that the inflammation pattern can also be shaped by lifestyle and environmental exposure with a trend toward a Th17-driven inflammation in presence of diesel particles ${ }^{(29)}$. A significant increase of ECP levels in tissue has also been revealed over the last 8 years in Southwest China, suggesting a shift to Th-2 eosinophilic inflammation over time in Asian countries ${ }^{(19)}$. One explanation could be a change in the nasal microbiome with an increase in the intramucosal carriage of Staphylococcus aureus ${ }^{(30)}$. Consequently, endotype-driven treatment options need to be customized geographically and may require adjustment with time. Further multicenter studies in larger cohorts of patients are also needed to confirm the diagnostic and predictive cut-off values of the above-mentioned markers. The cluster analysis considering biological markers together to define endotypes may provide a new area for defining CRSwNP diversity ${ }^{\left({ }^{8}\right)}$. In that regard, it will be important to set up the right combination of measurable biological indicators, while balancing the cost of testing and the required laboratory supplies (20).

\section{Conclusion}

Our study design was mainly focused on surgical failure defined as persistent symptoms after a first endoscopic sinus surgery in spite of a maximum medical treatment. Further studies should require a better analysis of quality of life with specific questionnaires (Sinonasal Outcome Test 22). With only eight patients eligible for a revision surgery in our study, a lack of statistical power cannot be excluded from our immune profile comparison. Nevertheless, with Th2 biomarkers measurement in nasal secretions, we provide a simple method of screening. It may allow us to identify CRSwNP patients with a higher risk for revision ESS after a first surgery, suggesting a smaller response to corticosteroids. This could be a step forward to properly select patients who will benefit the most from adjuvant anti-interleukins treatment.

\section{Acknowledgements}

Not applicable.

\section{Authorship contribution}

$\mathrm{GM}$ and $\mathrm{GL}$ contributed to the study conception and design. GM, CS, CC and IG were responsible for tissue sample and clinical data collection. Material preparation, data collection and analysis were performed by GM, IG and CS. The first draft of the manuscript was writtent by GM and IG. All authors commented on previous versions of the manuscript and approved the final submission.

\section{Conflict of interest}

The authors declare that they have no conflict of interest.

\section{References}

1. Fokkens WJ, Lund VJ, Mullol J, et al. European Position Paper on Rhinosinusitis and Nasal Polyps 2012. Rhinol Suppl. 2012;(23):3 p preceding table of contents, $1-298$.

2. De Greve G, Hellings PW, Fokkens WJ, Pugin B, Steelant B, Seys SF. Endotype-driven treatment in chronic upper airway diseases. Clin Transl Allergy. 2017;7:22.

3. Rudmik L. Economics of Chronic Rhinosinusitis. Curr Allergy Asthma Rep. 2017;17:20

4. Khan A, Huynh TMT, Vandeplas G, et al. The GALEN rhinosinusitis cohort: chronic rhinosinusitis with nasal polyps affects healthrelated quality of life. Rhinology. 2019 1;57:343-351.

5. Van Zele T, Claeys S, Gevaert P, et al. Differentiation of chronic sinus diseases by measurement of inflammatory mediators Allergy. 2006;61:1280-1289.

6. Van Zele T, Holtappels G, Gevaert P, Bachert C. Differences in initial immunoprofiles between recurrent and nonrecurrent chronic rhinosinusitis with nasal polyps. Am J Rhinol Allergy. 2014;28:192-198.

7. Borish L. Rethinking chronic rhinosinusitis phenotypes. J Allergy Clin Immunol. 2016;138:1354-1355.

8. Tomassen P, Vandeplas G, Van Zele T, et al Inflammatory endotypes of chronic rhinosinusitis based on cluster analysis of biomarkers. J Allergy Clin Immunol. 2016;137:14491456.e4.

9. DeConde AS, Mace JC, Levy JM, Rudmik L, Alt JA, Smith TL. Prevalence of polyp recurrence after endoscopic sinus surgery for chronic rhinosinusitis with nasal polyposis. Laryngoscope. 2017;127:550-555.
10. Ryu G, Kim DK, Dhong HJ, et al. Immunological Characteristics in Refractory Chronic Rhinosinusitis with Nasal Polyps Undergoing Revision Surgeries. Allergy Asthma Immunol Res. 2019;11:664-676.

11. Lildholdt T, Fogstrup J, Gammelgaard N, Kortholm B, Ulsoe C. Surgical versus medical treatment of nasal polyps. Acta Otolaryngol (Stockh). 1988;105:140-143.

12. Lund VJ, Mackay IS. Staging in rhinosinusitus. Rhinology. 1993;31:183-184.

13. Bachert C, Sousa AR, Lund VJ, et al. Reduced need for surgery in severe nasal polyposis with mepolizumab: Randomized trial. J Allergy Clin Immunol. 2017;140(4):10241031.e14.

14. Bachert C, Han JK, Desrosiers M, et al. Efficacy and safety of dupilumab in patients with severe chronic rhinosinusitis with nasal polyps (LIBERTY NP SINUS-24 and LIBERTY 
NP SINUS-52): results from two multicentre, randomised, double-blind, placebo-controlled, parallel-group phase 3 trials. Lancet Lond Engl. 2019;394(10209):1638-1650.

15. Mortuaire G, Gengler I, Balden M, Capron M, Lefèvre G. Impact of allergy on phenotypic and endotypic profiles of nasal polyposis. Eur Ann Otorhinolaryngol Head Neck Dis. 2018;135:159-162.

16. Mortuaire G, Gengler I, VandenhendeSzymanski C, Delbeke M, Gatault S, Chevalier D, et al. Immune profile modulation of blood and mucosal eosinophils in nasal polyposis with concomitant asthma. Ann Allergy Asthma Immunol Off Publ Am Coll Allergy Asthma Immunol. 2015:114:299-307.e2.

17. Turner JH, Li P, Chandra RK. Mucus T helper 2 biomarkers predict chronic rhinosinusitis disease severity and prior surgical intervention. Int Forum Allergy Rhinol. 2018;8:11751183.

18. Mendelsohn D, Jeremic G, Wright ED, Rotenberg BW. Revision rates after endoscopic sinus surgery: a recurrence analysis. Ann Otol Rhinol Laryngol. 2011;120:162166.

19. Wei B, Liu F, Zhang J, Liu Y, et al. Multivariate analysis of inflammatory endotypes in recurrent nasal polyposis in a Chinese population. Rhinology. 2018;56:216-226.

20. Lou H, Zhang N, Bachert C, Zhang L. Highlights of eosinophilic chronic rhinosinusitis with nasal polyps in definition, prog nosis, and advancement. Int Forum Allergy Rhinol. 2018;8:1218-1225.
21. Lou H, Meng Y, Piao Y, Wang C, Zhang L, Bachert C. Predictive significance of tissue eosinophilia for nasal polyp recurrence in the Chinese population. Am J Rhinol Allergy. 2015;29:350-356.

22. Tokunaga T, Sakashita M, Haruna T, Asaka D, Takeno S, Ikeda H, et al. Novel scoring system and algorithm for classifying chronic rhinosinusitis: the JESREC Study. Allergy. 2015;70:995-1003.

23. De Schryver E, Devuyst L, Derycke L, et al. Local immunoglobulin e in the nasal mucosa: clinical implications. Allergy Asthma Immunol Res. 2015;7:321-331.

24. Seshadri S, Rosati M, Lin DC, et al. Regional differences in the expression of innate host defense molecules in sinonasal mucosa. J Allergy Clin Immunol. 2013;132:1227-1230. e5.

25. Oyer SL, Mulligan JK, Psaltis AJ, Henriquez OA, Schlosser RJ. Cytokine correlation between sinus tissue and nasal secretions among chronic rhinosinusitis and controls. Laryngoscope. 2013;123:E72-78.

26. Wang $X$, Zhang N, Bo M, et al. Diversity of $\mathrm{TH}$ cytokine profiles in patients with chronic rhinosinusitis: A multicenter study in Europe, Asia, and Oceania. J Allergy Clin Immunol. 2016;138:1344-1353.

27. Bachert C, Zhang N, Holtappels G, et al. Presence of IL-5 protein and IgE antibodies to staphylococcal enterotoxins in nasal polyps is associated with comorbid asthma. J Allergy Clin Immunol. 2010;126:962-968, 968.e1-6.

28. Turner JH, Chandra RK, Li P, Bonnet K,
Schlundt DG. Identification of clinically relevant chronic rhinosinusitis endotypes using cluster analysis of mucus cytokines. J Allergy Clin Immunol. 2018;141:1895-1897. e7.

29. van Voorhis M, Knopp S, Julliard W, et al. Exposure to atmospheric particulate matter enhances Th17 polarization through the aryl hydrocarbon receptor. Plos One. 2013;8:e82545.

30. Katotomichelakis M, Tantilipikorn $P$, Holtappels G, et al. Inflammatory patterns in upper airway disease in the same geographical area may change over time. Am J Rhinol Allergy. 2013;27:354-60.

\section{Geoffrey Mortuaire}

Service d'ORL de chirurgie cervico-

-faciale

Hôpital Huriez

Rue Michel Polonovski

CHU Lille

59037 Lille Cedex

France

Tel: (+33) 320445675

Fax: (+33) 320446220

E-mail:

geoffrey.mortuaire@chru-lille.fr 